\title{
NONLINEAR RANDOM OPERATOR EQUATIONS AND INEQUALITIES IN BANACH SPACES
}

\author{
ANTONIOS KARAMOLEGOS \\ and \\ DIMITRIOS KRAVVARITIS \\ Department of Mathematics \\ National Technical University of Athens \\ Zografou Campus, 15773 \\ Athens, Greece
}

(Received July 31, 1989 and in revised form May 5, 1990)

\begin{abstract}
In this paper we give some new existence theorems for nonlinear random equations and inequalities involving operators of monotone type in Banach spaces. A random Hammerstein integral equation is also studied. In order to obtain random solutions we use some results from the existing deterministic theory as well as from the theory of measurable multifunctions and, in particular, the measurable selection theorems of Kuratowski/Ryll-Nardzewski and of Saint-Beuve.
\end{abstract}

KEY WORDS AND PHRASES. Random equations and inequalities, operators of monotone type, coercive operators, measurable multifunctions.

1991 AMS SUBJECT CLASSIFICATION CODES. 47H05, $60 \mathrm{H} 99$.

1. INTRODUCTION.

In recent years the theory of random nonlinear operator equations has attracted the attention of many authors (e.g. Engl [1], Itoh [2,3], Kravvaritis [4,5], Papageorgiou [6]). This interest is partly due to the fact that there are many applications of this theory to various applied fields such as control theory, statistics, biological sciences and others. For a discussion of such applications one may consult the books by Bharucha-Reid [7] and Padgett/Tsokos [8]. In this paper we present some new existence theorems for solutions to random nonlinear operator equations and inequalities. To obtain them we use some results from the existing deterministic theory as well as from the theory of measurable multifunctions and, in particular, the measurable selection theorems of Kuratowski/Ryll-Nardzewski and of Sainte-Beuve.

In Section 2, we fix our notation and recall some basic definitions from the theory of measurable multifunctions and from nonlinear functional analysis.

In Section 3, we give first a random fixed point theorem and then a perturbation result concerning operators of monotone type.

In Section 4, we treat random nonlinear variational inequalities. Two of them involve random operators of monotone type and a random convex function and one concerns a multivalued operator that does not satisfy any monotonicity condition.

Finally, in Section 5, a concrete random Hammerstein integral equation is studied.

2. PRELIMINARIES.

Let $(\Omega, \Sigma)$ be a measurable space and $X$ a separable Banach space. Let $F: \Omega \rightarrow 2^{X} \backslash\{\phi\}$ be a multifunction with closed values. We say that $F$ is measurable if it satisfies the following equivalent conditions: 
(i) For all $U \subseteq X$ open, $F^{-}(U)=\{\omega \in \Omega: F(\omega) \cap U \neq \emptyset\} \in \Sigma$,

(ii) There exist $f_{n}: \Omega \rightarrow X$, measurable for all $n \in \mathbb{N}$, such that:

$F(\omega)=\operatorname{cl}\left\{f_{n}(\omega)\right\}_{n \in N}$ for all $\omega \in \Omega$ (Castaing representation).

If, in addition, there exists a complete, $\sigma$-finite measure $\mu$, defined on $\Sigma$, then (i) and (ii) are equivalent to:

(iii) $\operatorname{Gr} F=\{(\omega, x) \in \Omega \times X: x \in F(\omega)\} \in \Sigma \otimes B(X)$, where $B(X)$ is the Borel $\sigma$-algebra of $X$.

Let $X$ be a reflexive Banach space and $X^{*}$ its topological dual. By (.,.) we denote the duality pairing between $X$ and $X^{*}$. The duality mapping $J$ of $X$ into $X^{*}$ is defined by:

$$
J x=\left\{x^{*} \in X^{*}:\left(x^{*}, x\right)=\|x\|^{2} \text { and }\left\|x^{*}\right\|=\|x\|\right\} .
$$

The symbols " $\rightarrow$ " and " $\stackrel{w}{\rightarrow}$ " denote convergence in the strong and weak topology, respectively.

An operator $T: D \subseteq X \rightarrow X^{*}$ is called (a) demicontinuous if $x_{n} \in D$ and $x_{n} \rightarrow x \in D$ imply $T x_{n} \stackrel{w}{\rightarrow} T x$, (b) weakly continuous if $x_{n} \in D$ and $x_{n} \stackrel{w}{\rightarrow} x \in D$ imply $T x_{n} \stackrel{w}{\rightarrow} T x$, (c) weakly closed if $x_{n} \in D, \quad x_{n} \stackrel{w}{\rightarrow} x \in D$ and $T x_{n} \stackrel{w}{\rightarrow} y$ imply $y=T x$, (d) quasi-bounded if to each $M>0$ there corresponds a constant $K(M)$ such that whenever $x \in D,(T x, x) \leq M\|x\|$ and $\|x\| \leq M$ it follows that $\|T x\| \leq K(M)$, (e) monotone if $(T x-T y, x-y) \geq 0$ for all $x, y \in D$, (f) maximal monotone if it is monotone and there is no proper extension of $T$ that is also a monotone operator, (g) pseudomonotone if ( $D$ is a closed, convex subset of $X$ ) the following conditions are satisfied: (P1) For any finite dimensional subspace $F$ of $X, T$ is continuous from $F \cap D$ into $X^{*}$, endowed with the weak topology; (P2) If $x_{n} \in D, x_{n} \stackrel{w}{\rightarrow} x \in D, T x_{n} \stackrel{w}{\rightarrow} f$ and $\lim \sup \left(T x_{n}, x_{n}-x\right) \leq 0$ then $f=T x$ and $\lim \left(T x_{n}, x_{n}\right)=(f, x), \quad(\mathrm{h})$ of type $\underline{(\mathrm{M})}$ if $x_{n} \in D, x_{n} \stackrel{w}{\rightarrow} x \in D, T x_{n} \stackrel{w}{\rightarrow} f$ and $\limsup \left(T x_{n}, x_{n}-x\right) \leq 0$ imply that $f=T x$.

For an operator $T: \Omega \times D \rightarrow X^{*}$ we will write $T(\omega) x$ for the value of $T$ at $(\omega, x) \in \Omega \times D$. Then $T$ is called random, if, for any $x \in D, T(\cdot) x$ is measurable. A random operator $T$ is called coercive if there exists a function $c: \mathbf{R}^{+} \rightarrow \mathbf{R}$, with $c(r) \rightarrow+\infty$ as $r \rightarrow+\infty$, such that

$$
(T(\omega) x, x) \geq c(\|x\|) \cdot\|x\| \text { for all } \omega \in \Omega \text { and } x \in D .
$$

A random operator $T$ is said to be monotone, demicontinuous, etc., if, for every $\omega \in \Omega, T(\omega)(\cdot)$ is monotone, demicontinuous, etc. We symbolize by $B(\Omega, X)$ the set of measurable functions $\xi: \Omega \rightarrow X$ such that $\sup \{\|\xi(\omega)\|: \omega \in \Omega\}<\infty$.

\section{RANDOM EQUATIONS.}

We begin with a random fixed point theorem which generalizes Theorem 6 of Ricceri [9]. In this and in the following sections we fix $(\Omega, \Sigma, \mu)$ to denote a complete, $\sigma$-finite, measure space.

THEOREM 3.1. Let $H$ be a separable Hilbert space and $D$ a convex, closed and bounded subset of $H$ with $0 \in \operatorname{int} D$. Let $A: \Omega \times D \rightarrow H$ be a random and weakly continuous operator such that $(A(\omega) x, x) \leq\|x\|^{2}$ for all $x \in \partial D$ and $\omega \in \Omega$. Then $A$ admits a random fixed point, i.e., there exists a measurable $\xi: \Omega \rightarrow D$ such that $A(\omega) \xi(\omega)=\xi(\omega)$ for all $\omega \in \Omega$.

PROOF. For every $\omega \in \Omega$, we define a mapping $R: \Omega \rightarrow 2^{D}$ by $R(\omega)=\{x \in D: x=A(\omega) x\}$. By Theorem 6 of Ricceri [9] we have $R(\omega) \neq \emptyset$ for all $\omega \in \Omega$. Let $\left\{y_{n}\right\}_{n \in N}$ be a dense sequence into $H$. We note that

$$
\operatorname{Gr} R=\{(\omega, x) \in \Omega \times D: x-A(\omega) x=0\}=\bigcap_{n=1}^{\infty}\left\{(\omega, x):\left(x-A(\omega) x, y_{n}\right)=0\right\}
$$

Now, for each $n \in \mathbb{N}$, the mapping $f_{n}: \Omega \times D \rightarrow \mathbb{R}$ defined by $f_{n}(\omega, x)=\left(x-A(\omega) x, y_{n}\right)$ is measurable in $\omega$ and continuous in $x$. By [10, Theorem 6.1] $f_{n}$ is jointly measurable, so Gr $R \in \Sigma \otimes B(D)$. Applying Theorem 3 of Sainte-Beuve [11], there exists a measurable selection of $R$, i.e., a measurable $\xi: \Omega \rightarrow D$ such that

$$
A(\omega) \xi(\omega)=\xi(\omega) \quad \text { for all } \omega \in \Omega .
$$

We study now perturbations of random maximal monotone operators by random operators of type 
(M). The deterministic case of the following theorem has been obtained in [12].

THEOREM 3.2. Let $X$ be a separable, reflexive Banach space and $D_{1}, D_{2}$ subsets of $X$. Let $L: \Omega \times D_{1} \rightarrow X^{*}$ be a jointly measurable, maximal monotone and weakly closed operator with $L(\omega) 0=0$ for all $\omega \in \Omega$. Also let $T: \Omega \times D_{2} \rightarrow X^{*}$ be a random, quasi-bounded, coercive and of type $(M)$ operator. Suppose that there exists a dense linear subspace $X_{0}$ of $X$, which is contained in $D_{2}$, such that for each finite dimensional subspace $F$ of $X_{0}$, the operator $T: \Omega \times F \rightarrow X^{*}$ is demicontinuous. Then, for each $y \in B\left(\Omega, X^{*}\right)$ there exists $x \in B(\Omega, X)$ such that:

$$
L(\omega) x(\omega)+T(\omega) x(\omega)=y(\omega) \quad \text { for all } \omega \in \Omega .
$$

PROOF. We may assume, without loss of generality, that $y(\omega)=0$ for all $\omega \in \Omega$. Also, by a result of Trojanski, we may suppose that the spaces $X$ and $X^{*}$ are locally uniformly convex. Thus, the mapping $J^{-1}: X^{*} \rightarrow X$ is continuous from the strong topology of $X^{*}$ to the strong topology of $X$ (cf. [13]). For $\varepsilon>0$ and $\omega \in \Omega$, let $L_{\varepsilon}(\omega)$ be the Yosida approximant of $L(\omega)$ defined by:

$$
L_{\varepsilon}(\omega) x=\left(L(\omega)^{-1}+\varepsilon J^{-1}\right)^{-1} x .
$$

$L_{\varepsilon}(\omega)$ is everywhere defined, single valued, bounded, maximal monotone with $L_{\varepsilon}(\omega) 0=0$ (cf. [13]).

We show that $L_{\varepsilon}: \Omega \times X \rightarrow X^{*}$ is random. Fix $x \in X$ and consider

$$
\begin{aligned}
\operatorname{Gr} L_{\varepsilon}(\cdot) x & =\left\{(\omega, y) \in \Omega \times X^{*}: y=\left(L(\omega)^{-1}+\varepsilon J^{-1}\right)^{-1} x\right\}=\left\{(\omega, y): x \in L(\omega)^{-1} y+\in J^{-1} y\right\} \\
& =\left\{(\omega, y):\left(x-\varepsilon J^{-1} y\right) \in L(\omega)^{-1} y\right\}=\left\{(\omega, y): y=L(\omega)\left(x-\varepsilon J^{-1} y\right)\right\} \\
& =\left\{(\omega, y): L(\omega)\left(x-\varepsilon J^{-1} y\right)-y=0\right\} .
\end{aligned}
$$

Now, the mapping $y \rightarrow x-\varepsilon J^{-1} y$, being continuous, is measurable, so $(\omega, y) \rightarrow\left(\omega, x-\varepsilon J^{-1} y\right)$ is measurable. Composing this and $L$ we get that $(\omega, y) \rightarrow L(\omega)\left(x-\varepsilon J^{-1} y\right)$ is measurable. Finally, $(\omega, y) \rightarrow L(\omega)\left(x-\varepsilon J^{-1} y\right)-y$ is measurable and thus $\operatorname{Gr} L_{\varepsilon}(\cdot) \in \Sigma \otimes B\left(X^{*}\right)$. Since $(\Omega, \Sigma, \mu)$ is complete, by Himmelberg [10, Theorem 3.4], $L_{\varepsilon}(\cdot) x$ is measurable, i.e., $L_{\varepsilon}$ is random.

Let $\left\{X_{n}\right\}_{n \in N}$ be an increasing sequence of finite dimensional subspaces of $X_{0}$, such that $\bigcup_{n=1}^{\infty} X_{n}$ is dense in $X$. For each $n \in \mathbb{N}$, let $j_{n}$ be the injection mapping of $X_{n}$ into $X$ and $j_{n}^{*}$ its dual. Clearly, the operator $K_{n \varepsilon}: \Omega \times X_{n} \rightarrow X_{n}^{*}$ defined by $K_{n \varepsilon}(\omega) x=j_{n}^{*}\left(L_{\varepsilon}(\omega)+T(\omega)\right) j_{n} x$ is random and continuous. Since $L_{\varepsilon}(\omega) 0=0$ and $T$ is coercive, $K_{n \varepsilon}$ is also coercive.

By Itoh [2, Proposition 3.1] there exists a measurable mapping $x_{n \varepsilon}: \Omega \rightarrow X_{n}$ such that $K_{n \varepsilon}(\omega) x_{n \varepsilon}(\omega)=0$ for all $\omega \in \Omega$. Now, we have:

$$
0=\left(L_{\varepsilon}(\omega) x_{n \varepsilon}(\omega)+T(\omega) x_{n \varepsilon}(\omega), x_{n \varepsilon}(\omega) \geq\left(T(\omega) x_{n \varepsilon}(\omega), x_{n \varepsilon}(\omega) \geq c\left(\left\|x_{n \varepsilon}(\omega)\right\|\right) \cdot\left\|x_{n \varepsilon}(\omega)\right\| .\right.\right.
$$

It follows from the growth property of $c(r)$ that there exists a constant $M>0$ such that $\left\|x_{n \varepsilon}(\omega)\right\| \leq M \quad$ for all $n \in \mathbb{N}, \quad \varepsilon>0$ and $\omega \in \Omega$. We set $u_{n \varepsilon}(\omega)=L_{\varepsilon}(\omega) x_{n \varepsilon}(\omega)$ and $v_{n \varepsilon}(\omega)=T(\omega) x_{n \varepsilon}(\omega)$. Because $L_{\varepsilon}$ is bounded and $T$ quasi-bounded the sequences $\left\{u_{n \varepsilon}(\omega)\right\}$ and $\left\{v_{n \varepsilon}(\omega)\right\}$ are bounded, therefore $\left\{u_{n \varepsilon}(\omega)+v_{n \varepsilon}(\omega)\right\}$ is bounded and, since $\bigcup_{n=1}^{\infty} X_{n}$ is dense in $X$, we get that

$$
u_{n \varepsilon}(\omega)+v_{n \varepsilon}(\omega) \stackrel{w}{\rightarrow} 0 \text { in } X^{*}, \text { as } n \rightarrow \infty .
$$

Let $\Gamma_{n \varepsilon}(\omega)=$ weakcl $\left\{x_{i \varepsilon}(\omega): i \geq n\right\}$, for every $n \in \mathbb{N}$. Under the weak topology $B_{M}(0)=\{x \in X:\|x\| \leq M\}$ is a metrizable space. Thus, by [10, Theorem 5.6] the multifunctions $\Gamma_{n \varepsilon}$ are weakly measurable. Then the multifunction $\Gamma_{\varepsilon}: \Omega \rightarrow 2{ }^{B} M^{(0)}$ defined by

$$
\Gamma_{\varepsilon}(\omega)=\bigcap_{n=1}^{\infty} \Gamma_{n \varepsilon}(\omega), \quad \text { for each } \omega \in \Omega
$$

is also weakly measurable [10, Theorem 4.1]. By the well-known theorem of Kuratowski and RyllNardzewski there is a weakly measurable selector $x_{\varepsilon}: \Omega \rightarrow B_{M}(0)$ of $\Gamma_{\varepsilon}$. Because of the separability 
of $X, x_{\varepsilon}$ is also measurable when $B_{M}(0)$ has the norm topology. For a fixed $\omega \in \Omega$, there is a subsequence of $\left\{x_{n \varepsilon}(\omega)\right\}$ (denoted again by $\left\{x_{n \varepsilon}(\omega)\right\}$ ) such that

$$
x_{n \varepsilon}(\omega) \stackrel{w}{\rightarrow} x_{\varepsilon}(\omega), \text { as } n \rightarrow \infty
$$

Besides, we may assume that $u_{n \varepsilon}(\omega) \stackrel{w}{\rightarrow} u_{\varepsilon}(\omega)$ and $v_{n \varepsilon}(\omega) \stackrel{w}{\rightarrow} v_{\varepsilon}(\omega)$ as $n \rightarrow \infty$.

It is clear that

$$
u_{\varepsilon}(\omega)+v_{\varepsilon}(\omega)=0 \quad \text { for all } \omega \in \Omega
$$

Let $\left\{\varepsilon_{\kappa}\right\}_{\kappa \in N}$ be a sequence of positive numbers such that $\varepsilon_{\kappa} \rightarrow 0$, as $\kappa \rightarrow \infty$. We set $x_{\kappa}(\omega)=x_{\varepsilon_{\kappa}}(\omega)$ and

$$
R(\omega)=\bigcap_{\kappa=1}^{\infty} \text { weakcl }\left\{x_{i}(\omega): i \geq \kappa\right\}
$$

As before, we deduce that there exists a measurable mapping $x: \Omega \rightarrow X$ such that $x(\omega) \in R(\omega)$ for all $\omega \in \Omega$. For a fixed $\omega \in \Omega$, there exists a subsequence of $\left\{x_{\kappa}(\omega)\right\}$ (which, we again denote by $\left.\left\{x_{\kappa}(\omega)\right\}\right)$ such that

$$
x_{\kappa}(\omega) \stackrel{w}{\rightarrow} x(\omega), \quad u_{\kappa}(\omega)=u_{\varepsilon_{\kappa}}(\omega) \stackrel{w}{\rightarrow} u(\omega) \text { and } y_{\kappa}(\omega)=v_{\varepsilon_{\kappa}}(\omega) \stackrel{w}{\rightarrow} v(\omega), \text { as } \kappa \rightarrow \infty
$$

Clearly,

$$
u(\omega)+v(\omega)=0 \quad \text { for all } \omega \in \Omega
$$

As in the deterministic case [12], one can prove that

$$
u(\omega)=L(\omega) x(\omega) \text { and } v(\omega)=T(\omega) x(\omega)
$$

So,

$$
L(\omega) x(\omega)+T(\omega) x(\omega)=0 \quad \text { for all } \omega \in \Omega
$$

i.e., $x(\cdot)$ is the desired solution.

REMARK. The assumptions on $L$ are satisfied when $L$ is random, weakly continuous, maximal monotone and $L(\omega) 0=0$ for all $\omega \in \Omega$. (In particular, when $L$ is random, linear, maximal monotone, with $D_{1}=X$ ). Then, clearly, $L$ is weakly closed. In addition, $L$ is jointly measurable. Indeed, if $y$ is any element of $X$, the operator $(\omega, x) \rightarrow(L(\omega) x, y)$ is a Caratheodory function, hence measurable. It follows that $L$ is weakly jointly measurable and, since $X^{*}$ is separable, $L$ is also jointly measurable.

\section{NONLINEAR RANDOM INEQUALITIES.}

The theorem which follows gives a random version of Theorem 4 in [9].

THEOREM 4.1. Let $X$ be a separable, reflexive Banach space, $D$ a convex, closed subset of $X$ with $\operatorname{int}_{\operatorname{Aff}(D)} D \neq \emptyset$ (i.e. the interior of $D$, relative to the affine space generated by $D$, is nonempty). If $\Phi: \Omega \times D \rightarrow X^{*}$ is a multifunction such that:

1) $\Phi(\omega) x$ is non-empty, convex and weakly compact subset of $X^{*}$ for all $\omega \in \Omega$ and $x \in D$.

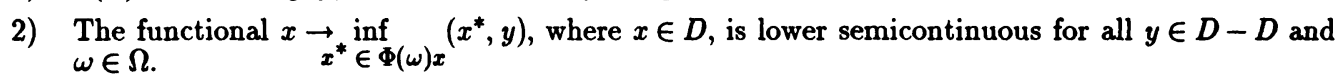

3) There exists a compact set $K \subseteq D$ and a point $y_{0} \in K$ with the property $\inf _{x^{*}} \in \Phi(\omega) x$
for all $x \in D \backslash \mathrm{K}$ and $\omega \in \Omega$.

4) The graph of the multifunction $\left.\Phi\right|_{\Omega \times K}$ (restriction of $\Phi$ to $\Omega \times K$ ) belongs to $\Sigma \otimes B(K) \otimes B\left(X^{*}\right)$.

Then there exist measurable mappings $\xi: \Omega \rightarrow K$ and $\eta: \Omega \rightarrow X^{*}$ such that, for all $\omega \in \Omega$,

$$
\eta(\omega) \in \Phi(\omega) \xi(\omega) \text { and }(\eta(\omega), \xi(\omega)-y) \leq 0 \text { for all } y \in D \text {. }
$$


PROOF. We consider the multifunction $F: \Omega \rightarrow K \times X^{*}$ defined by

$$
F(\omega)=\left\{(x, z) \in K \times X^{*}: z \in \Phi(\omega) x \text { and }(z, x-y) \leq 0 \text { for all } y \in D\right\}
$$

By Theorem 4 of Ricceri [9], we have $R(\omega) \neq \emptyset$ for all $\omega \in \Omega$. Let $\left\{y_{n}\right\}_{n \in N}$ be a sequence of points of $D$, dense into $D$. Note that:

$$
F(\omega)=\bigcap_{n=1}^{\infty}\left\{(x, z) \in K \times X^{*}: z \in \Phi(\omega) x \text { and }\left(z, x-y_{n}\right) \leq 0\right\}
$$

Defining $F_{n}: \Omega \rightarrow K \times X^{*}$ by

$$
F_{n}(\omega)=\left\{(x, z) \in K \times X^{*}: z \in \Phi(\omega) x \text { and }\left(z, x-y_{n}\right) \leq 0\right\}
$$

we have that $\operatorname{Gr} F=\bigcap_{n=1}^{\infty} \operatorname{Gr} F_{n}$ and that

$$
\begin{aligned}
\operatorname{Gr} F_{n} & =\left\{(\omega, x, z) \in \Omega \times K \times X^{*}: z \in \Phi(\omega) x \text { and }\left(z, x-y_{n}\right) \leq 0\right\} \\
& =\left.\left\{(\omega, x, z) \in \Omega \times K \times X^{*}:\left(z, x-y_{n}\right) \leq 0\right\} \cap \operatorname{Gr} \Phi\right|_{\Omega \times K}
\end{aligned}
$$

Because $K$ and $X^{*}$ are Suslin spaces, it holds $B\left(K \times X^{*}\right)=B(K) \otimes B\left(X^{*}\right)$. The set $\left\{(\omega, x, z) \in \Omega \times K \times X^{*}:\left(z, x-y_{n}\right) \leq 0\right\}$, being equal to $\Omega \times\left\{(x, z) \in K \times X^{*}:\left(z, x-y_{n}\right) \leq 0\right\}$, clearly belongs to $\Sigma \otimes B\left(K \times X^{*}\right)$. By assumption, $\left.\operatorname{Gr} \Phi\right|_{\Omega \times K}$ belongs to $\Sigma \otimes B(K) \otimes B\left(X^{*}\right)=\Sigma \otimes B\left(K \times X^{*}\right)$. So $\operatorname{Gr} F_{n} \in \Sigma \otimes B\left(K \times X^{*}\right)$ which in turn implies $\operatorname{Gr} F \in \Sigma \otimes B\left(K \times X^{*}\right)$. By the selection theorem of Sainte-Beuve, there exists a measurable mapping $h: \Omega \rightarrow K \times X^{*}$ such that $h(\omega) \in F(\omega)$ for all $\omega \in \Omega$. If we put $h=(\xi, \eta)$ it follows that $\xi: \Omega \rightarrow K$ and $\eta: \Omega \rightarrow X^{*}$ are measurable mappings, that $\eta(\omega) \in \Phi(\omega) \xi(\omega)$ for all $\omega \in \Omega$ and that

$$
(\eta(\omega), \xi(\omega)-y) \leq 0 \quad \text { for all } y \in D \text { and } \omega \in \Omega .
$$

We shall need the concept of separability for a random function $\varphi: \Omega \times X \rightarrow \mathbb{R}$, where $X$ is a separable, metrizable and complete space (cf. Bharucha-Reid [7]).

Separable random functions are characterized in the following way (for the proof, see Papageorgiou [14]): A random function $\varphi: \Omega \times X \rightarrow R$ is separable, if, and only if, there exists a countable, dense set $D \subseteq X$ and a $N \in \Sigma, \mu(N)=0$, such that for $\omega \notin N$ and for $x \in X$, there exists a sequence $x_{n} \in D$, such that $x_{n} \rightarrow x$ and $\varphi\left(\omega, x_{n}\right) \rightarrow \varphi(\omega, x)$.

THEOREM 4.2. Let $X$ be a separable, reflexive Banach space and $K$ a closed, convex and bounded subset of $X$. If $T: \Omega \times K \rightarrow X^{*}$ is a random, monotone and demicontinuous operator and $\varphi: \Omega \times K \rightarrow \mathbb{R}$ is a random, convex, lower semicontinuous and separable function, then for each measurable $y: \Omega \rightarrow X^{*}$ there exists a measurable $x: \Omega \rightarrow K$ such that

$$
(T(\omega) x(\omega)-y(\omega), x(\omega)-z) \leq \varphi(\omega, z)-\varphi(\omega, x(\omega)) \quad \text { for all } z \in K \text { and for almost all } \omega \in \Omega .
$$

PROOF. We may assume that $y(\omega)=0$ for all $\omega \in \Omega$. We consider the multifunction $R: \Omega \rightarrow 2^{K}$ defined by

$$
R(\omega)=\{x \in K:(T(\omega) x, x-z) \leq \varphi(\omega, z)-\varphi(\omega, x) \text { for all } z \in K\} .
$$

From Browder [15], we have $R(\omega) \neq \emptyset$ for all $\omega \in \Omega$. Let $D=\left\{x_{n}\right\}_{n \in N}$ be a countable dense subset of $K$ and $N$ the $\mu$-null set postulated by the separability of $\varphi$. Then, for all $\omega \in \Omega \backslash N$, we have

$$
R(\omega)=\bigcap_{n=1}^{\infty}\left\{x \in K:\left(T(\omega) x, x-x_{n}\right) \leq \varphi\left(\omega, x_{n}\right)-(\omega, x)\right\}
$$

We set $R_{n}(\omega)=\left\{x \in K:\left(T(\omega) x, x-x_{n}\right) \leq \varphi\left(\omega, x_{n}\right)-\varphi(\omega, x)\right\}$.

By Theorem 3.1 of Papageorgiou [14] the mapping $(\omega, x) \rightarrow\left(T(\omega) x, x-x_{n}\right)-\varphi\left(\omega, x_{n}\right)+\varphi(\omega, x)$ from $(\Omega \backslash N) \times K$ to $\mathbb{R}$ is jointly measurable. So, Gr $R_{n} \in \Sigma_{\Omega \backslash N} \otimes B(K)$ and, consequently, $\operatorname{Gr} R=\bigcap_{n=1}^{\infty} \operatorname{Gr} R_{n} \in \Sigma_{\Omega \backslash N} \otimes B(K)$. Since the measure space $\left(\Omega \backslash N, \Sigma_{\Omega \backslash N}, \mu\right)$ is complete, applying 
Sainte-Beuve's selection theorem, we get measurable $x: \Omega \backslash N \rightarrow K$ such that $x(\omega) \in R(\omega)$ for all $\omega \in \Omega \backslash N$, i.e.,

$$
(T(\omega) x(\omega), \quad x(\omega)-z) \leq \varphi(\omega, z)-\varphi(\omega, x(\omega)) \quad \text { for all } z \in K \text { and almost all } \omega \in \Omega .
$$

Now we prove an existence theorem for random inequalities involving a pseudomonotone operator $T$ and a random, continuous, convex function $\varphi$. It would be interesting to prove this theorem with the same assumptions on $\varphi$ as in the previous theorem.

THEOREM 4.3. If $T: \Omega \times K \rightarrow X^{*}$ is a random, pseudomonotone and bounded operator and $\varphi: \Omega \times K \rightarrow \mathbb{R}$ is a random, convex and continuous function, then for each measurable $y: \Omega \rightarrow X^{*}$ there exists a measurable $x: \Omega \rightarrow X$ such that $(T(\omega) x(\omega)-y(\omega), x(\omega)-z) \leq \varphi(\omega, z)-\varphi(\omega, x(\omega))$ for all $z \in K$ and $\omega \in \Omega$.

PROOF. We may assume that $y(\omega)=0$ for all $\omega \in \Omega$. Let $\left\{X_{n}\right\}_{n \in N}$ be an increasing sequence of finite dimensional subspaces of $X$, whose union is dense in $X$. We denote $j_{n}: X_{n} \rightarrow X$, the injection mapping of $X_{n}$ into $X$, and $j_{n}^{*}: X^{*} \rightarrow X_{n}^{*}$, its adjoint. We then define $T_{n}: \Omega \times K_{n} \rightarrow X_{n}^{*}$, where $K_{n}=K \cap X_{n}$, by $T_{n}(\omega) x=j_{n}^{*} T(\omega) j_{n} x$.

We consider the multifunction $R_{n}(\omega)=\left\{x \in K_{n}:\left(T_{n}(\omega) x, x-z\right) \leq \varphi(\omega, z)-\varphi(\omega, x)\right.$ for all $\left.z \in K_{n}\right\}$. From Browder [15], we know that $R_{n}(\omega) \neq \emptyset$ for all $\omega \in \Omega$ and $n \in \mathbb{N}$. Let $\left\{z_{m}\right\}_{m \in N}$ be a dense sequence into $K_{n}$. We have $R_{n}(\omega)=\bigcap_{m=1}^{\infty}\left\{x \in K_{n}:\left(T_{n}(\omega) x, x-z_{m}\right) \leq \varphi\left(\omega, z_{m}\right)-\varphi(\omega, x)\right\}$.

As in the proof of the previous theorem, we deduce that $\operatorname{Gr} R_{n} \in \Sigma \otimes B\left(K_{n}\right)$ and, by SainteBeuve's selection theorem, there exists $x_{n}: \Omega \rightarrow K$, measurable, such that $x_{n}(\omega) \in R_{n}(\omega)$, for all $\omega \in \Omega$, i.e.

$$
\left(T(\omega) x_{n}(\omega), x_{n}(\omega)-z\right) \leq \varphi(\omega, z)-\varphi\left(\omega, x_{n}(\omega)\right) \quad \text { for all } z \in K_{n}
$$

Let $\Gamma_{n}: \Omega \rightarrow 2^{K}$ be defined by $\Gamma_{n}(\omega)=$ weakcl $\left\{x_{i}(\omega): i \geq n\right\}$, for each $n \in \mathbb{N}$, and let $\Gamma: \Omega \rightarrow 2^{K}$ be such that $\Gamma(\omega)=\bigcap_{n=1}^{\infty} \Gamma_{n}(\omega)$ for all $\omega \in \Omega$.

As in the proof of Theorem 3.2, we deduce the existence of a measurable mapping $x: \Omega \rightarrow K$ such that $x(\omega) \in \Gamma(\omega)$, for all $\omega \in \Omega$. Fix $\omega \in \Omega$. There exists a subsequence of $\left\{x_{n}(\omega)\right\}$, which we again denote by $\left\{x_{n}(\omega)\right\}$, such that $x_{n}(\omega) \stackrel{w}{\rightarrow} x(\omega) \in K$.

Since $T$ is bounded, we may assume (passing to a subsequence, if necessary) that $T(\omega) x_{n}(\omega) \stackrel{w}{\rightarrow} u(\omega)$. Now, take $z \in K=\operatorname{cl}\left(\bigcup_{n=1}^{\infty} K_{n}\right)$. On can easily verify that (4.1) implies

$$
\lim \sup \left(T(\omega) x_{n}(\omega), x_{n}(\omega)-z\right) \leq \varphi(\omega, z)-\varphi(\omega, x(\omega))
$$

Putting $z=x(\omega) \in K$ in (4.2) we get :

$$
\lim \sup \left(T(\omega) x_{n}(\omega), x_{n}(\omega)-x(\omega)\right) \leq 0 .
$$

The pseudomonotone property of $T(\omega)$ implies

$$
u(\omega)=T(\omega) x(\omega) \text { and }\left(T(\omega) x_{n}(\omega), \quad x_{n}(\omega)\right) \rightarrow(T(\omega) x(\omega), x(\omega))
$$

So, inequality (4.2) becomes:

$$
(T(\omega) x(\omega), x(\omega)-z) \leq \varphi(\omega, z)-\varphi(\omega, x(\omega)), \quad \text { for any } z \in K .
$$

REMARK. Our result generalizes Theorem 24 of [16].

\section{A RANDOM HAMMERSTEIN INTEGRAL EQUATION.}

We study now a random nonlinear Hammerstein integral equation of the form

$$
u(\omega, x)+\int_{A} K(\omega, x, y) f(\omega, y, u(\omega, y)) d y=w(\omega, x)
$$

where $\Omega$ and $A$ are $\sigma$-finite measure spaces. Without loss of generality, the function $w(\omega, x)$ may be 
taken to be zero. Let $1<p, q<\infty$, such that $\frac{1}{p}+\frac{1}{q}=1$.

THEOREM 5.1. Let $f: \Omega \times A \times \mathbb{R} \rightarrow \mathbb{R}$ be a function such that:

1) For all $u \in \mathbb{R}$ the function $(\omega, y) \rightarrow f(\omega, y, u)$ is measurable and for all $(\omega, y) \in \Omega \times A$ the function $u \rightarrow f(\omega, y, u)$ is continuous.

2) There exists functions $\alpha(\omega, y)$, with $\alpha(\omega, \cdot) \in L_{+}^{p}(A)$ for all $\omega \in \Omega$ and $b(\omega)>0$ such that:

$$
|f(\omega, y, u)| \leq \alpha(\omega, y)+b(\omega)|u|^{p-1} \quad \text { for all } \omega \in \Omega, y \in A, u \in \mathbf{R}
$$

3) For all $\omega \in \Omega$ and $y \in A, f(\omega, y, u)$ is strictly monotone increasing with respect to $u$, i.e., $u>u^{\prime} \Rightarrow f(\omega, y, u)>f\left(\omega, y, u^{\prime}\right)$.

4) There exist functions $c_{1}(\omega, y)$, with $c_{1}(\omega, \cdot) \in L^{1}(A)$ for all $\omega \in \Omega$ and $c_{2}(\omega)>0$ such that:

$$
f(\omega, y, u) u \geq c_{2}(\omega)|u|^{p}-c_{1}(\omega, y) \text { for all } \omega \in \Omega, y \in A, u \in \mathbf{R} .
$$

5) There exists a function $c: \mathbb{R}^{+} \rightarrow \mathbb{R}$, with $c(r) \rightarrow+\infty$ as $r \rightarrow+\infty$, such that:

And let $K: \Omega \times A \times A \rightarrow \mathbb{R}$ be a function such that

$$
\int_{A} f(\omega, y, u(y)) u(y) d y \geq c\left(\|u\|_{p}\right) \cdot\|u\|_{p} \quad \text { for all } u \in L^{p}(A) .
$$

6) $(\omega, x, y) \rightarrow K(\omega, x, y)$ is measurable.

7) The operator $v(\cdot) \rightarrow w(\cdot)=\int_{A} K(\omega, \cdot, y) v(y) d y$ maps $L^{q}(A)$ into $L^{p}(A)$ for all $\omega \in \Omega$.

8) $\iint_{A} K(\omega, x, y) v(y) v(x) d y d x \geq 0 \quad$ for all $\omega \in \Omega$.

Then there exists a function $u: \Omega \times A \rightarrow \mathbb{R}$, jointly measurable, with $u(\omega, \cdot) \in L^{p}(A)$ for a.a. (almost all) $\omega \in \Omega$, which satisfies (5.1) for a.a $\omega \in \Omega$ and a.a. $x \in A$.

PROOF. Consider the operator $N(\omega) u(\cdot)=f(\omega, \cdot, u(\cdot))$. For every $\omega \in \Omega$, we know, by assumptions 1) and 2), that $N(\omega)(\cdot)$ is a bounded, continuous operator from $L^{p}(A)$ into $L^{q}(A)$. Also, from 3) and 4), it follows (see Browder [17, Proposition 1]) that for each $\omega \in \Omega, N(\omega)(\cdot)$ is an operator of type $\left(S_{+}\right)$, hence, also, of type $(\mathrm{M})$.

Fix $u \in L^{p}(A)$ and consider the map $\omega \rightarrow N(\omega) u$. We have

$$
\left\{\omega \in \Omega:\|N(\omega) u\|_{q}<\varepsilon\right\}=\left\{\omega \in \Omega: \int_{A}|f(\omega, y, u(y))|^{q} d y<\varepsilon^{q}\right\}
$$

By assumption 1), the function $(\omega, y) \rightarrow f(\omega, y, u(y))$ is measurable, hence

$$
(\omega, y) \rightarrow|f(\omega, y, u(y))|^{q}
$$

is measurable and so, by Fubini's theorem, $\omega \rightarrow \int_{A}|f(\omega, y, u(y))|^{q} d y$ is also measurable. Therefore, the above set is measurable and this proves that $\omega \rightarrow N(\omega) u$ is measurable. So $N: \Omega \times L^{p}(A) \rightarrow L^{q}(A)$ is random.

Also, assumption 5$)$ means that, for all $\omega \in \Omega, N(\omega)(\cdot)$ is a coercive operator, i.e., $N$ is coercive.

Next, we consider the linear integral operator $B(\omega)(\cdot)$ defined by

$$
B(\omega) v(\cdot)=\int_{A} K(\omega, \cdot, y) v(y) d y .
$$

From assumption 7), for each $\omega \in \Omega, B(\omega)(\cdot)$ is an operator mapping $L^{q}(A)$ into $L^{p}(A)$. Hypothesis 8) means that $B(\omega)(\cdot)$ is positive, so, being linear, is also monotone. It follows that $B(\omega)(\cdot)$ is continuous.

Now fix $v \in L^{q}(A)$ and consider the mapping $\omega \rightarrow B(\omega) v$. We have

$$
\left\{\omega \in \Omega:\|B(\omega) v\|_{p}<\varepsilon\right\}=\left\{\omega \in \Omega: \int\left|\int_{A} K(\omega, x, y) v(y) d y\right|^{p} d x<\varepsilon^{p}\right\}
$$

By assumption 5), the function $(\omega, x, y) \rightarrow K(\omega, x, y) v(y)$ is measurable, therefore the same holds for 
and for

$$
(\omega, x) \rightarrow \int_{A} K(\omega, x, y) v(y) d y
$$

and, finally, for

$$
(\omega, x) \rightarrow\left|\int_{A} K(\omega, x, y) v(y) d y\right|^{p}
$$

$$
\omega \rightarrow \int_{A}\left|\int_{A} K(\omega, x, y) v(y) d y\right|^{p} d x .
$$

So, the above set is measurable and this proves that $\omega \rightarrow B(\omega) v$ is measurable, i.e.,

is random. Rewrite equation (5.1) as

$$
B: \Omega \times L^{q}(A) \rightarrow L^{p}(A)
$$

$$
u(\omega)+B(\omega) N(\omega) u(\omega)=0 .
$$

Invoking Theorem 3 in [4] we conclude that (5.2) has a solution $\widehat{u} \in B\left(\Omega, L^{p}(A)\right)$. By Theorem III-17 of Dunford Schwartz [18] we deduce that there exists $u: \Omega \times A \rightarrow \mathbf{R}$, jointly measurable such that $u(\omega, \cdot)=\widehat{u}(\omega)(\cdot)$ for a.a. $\omega \in \Omega$. So $u(\omega, \cdot) \in L^{p}(A)$ for a.a. $\omega \in \Omega$ and $u(\omega, x)$ satisfies (5.1) for a.a. $\omega \in \Omega$ and a.a. $x \in A$.

\section{REFERENCES}

1. ENGL. H.W., Random Fixed Point Theorems for Multi-valued Mappings, Pacific L. Math. $\underline{76}$ (1978), 351-360.

2. ITOH, S., Nonlinear Random Equations with Monotone Operators in Banach Spaces, Math. Ann. 236 (1978), 133-146.

3. ITOH, S., Random Fixed Point Theorems with an Application to Random Differential Equations in Banach Spaces, J. Math. Anal. Appl. 67 (1979), 261-273.

4. KRAVVARITIS, D., Nonlinear Random Operators of Monotone Type in Banach Space, J. Math. Anal. Appl. 78, (1980), 488-496.

5. KRAVVARITIS, D., Nonlinear Random Equations Involving Operators of Monotone Type, $\mathrm{J}$. Math. Anal. Appl. 114 (1986), 295-304.

6. PAPAGEORGIOU, N.S., Random Fixed Point Theorems for Measurable Multifunctions in Banach Spaces, Proc. Amer. Math. Soc. 97 (1986), 507-514.

7. BHARUCHA-REID, A.T., Random Integral Equations, Academic Press, New York (1972).

8. PADGETT, W. and TSOKOS, C., Random Integral Equations with Applications to Life Sciences and Engineering, Academic Press, New York (1976).

9. RICCERI, B., Un théorème d'existence pour les inéquations variationalles, C.R. Acad. Sc. Paris, t. 301, Série I, $\mathbf{n}^{\circ} 19,1985$.

10. HIMMELBERG, C.J., Measurable Relations, Fund. Math. 87 (1.975), 53-72.

11. SAINTE-BEUVE, M.F., On the Extension of von Neumann-Aumann's Theorem, J. Funct. Anal. 17 (1974), 112-129.

12. KRAVVARITIS, D., Nonlinear Equations and Inequalities in Banach Spaces, J. Math. Anal. Appl. 67 (1979), 205-214.

13. PASCALI, D. and SBURLAN, S., Nonlinear Mappings of Monotone Type, Editura Academiei, Bururesti, 1978.

14. PAPAGEORGIOU, N.S., On Measurable Multifunctions with Applications to Random Multivalued Equations, Math. Japonica, 32 (1987), 701-727.

15. BROWDER, F., Nonlinear Variational Inequalities and Maximal Monotone Mappings in Banach Spaces, Math. Ann. 183 (1969), 213-231.

16. BREZIS, H., Equations et Inéquations non Linéaires dans les espaces vectoriels en Dualité, Ann. Inst. Fourier, 18, 1 (1968), 115-175.

17. BROWDER, F., Nonlinear Functional Analysis and Nonlinear Integral Equations of Hammerstein and Urysohn Type, Contributions to Nonlinear functional analysis, 425500, Academic Press (1971).

18. DUNFORD, N. and SCHWARTZ, J.T., Linear Operators, I, Wiley, New York (1958). 


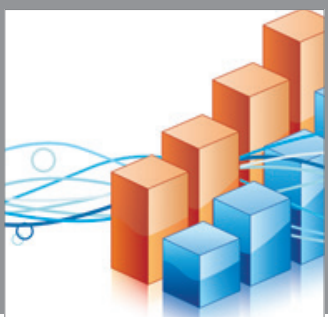

Advances in

Operations Research

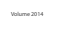

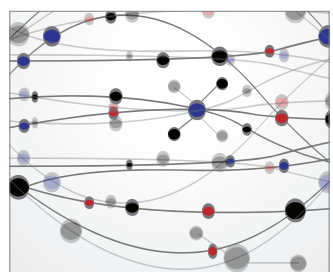

\section{The Scientific} World Journal
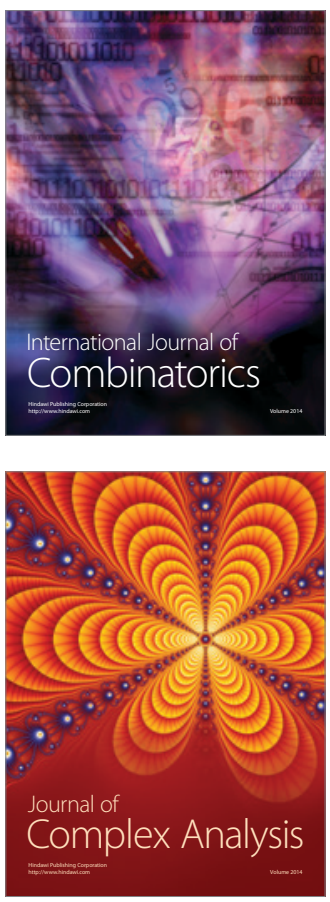

International Journal of

Mathematics and

Mathematical

Sciences
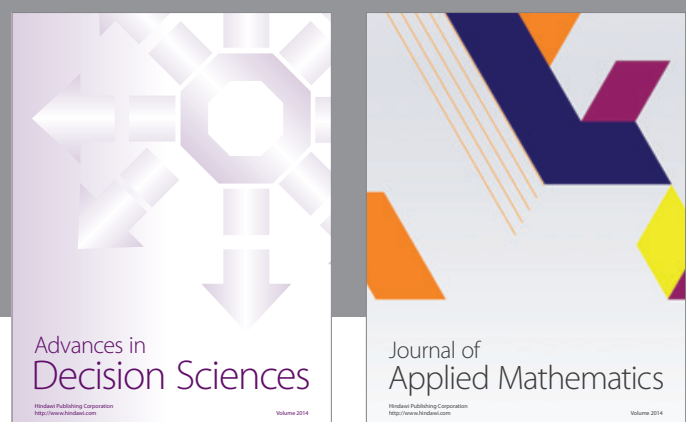

Journal of

Applied Mathematics
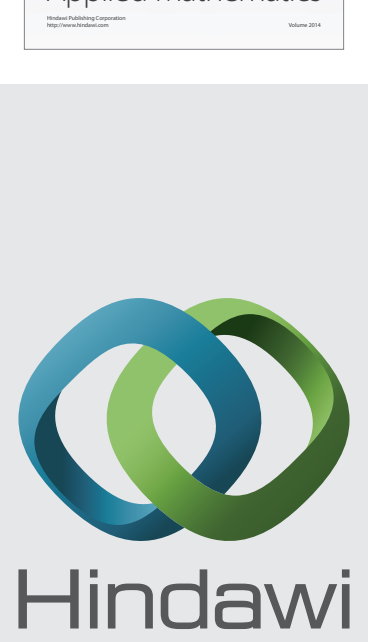

Submit your manuscripts at http://www.hindawi.com
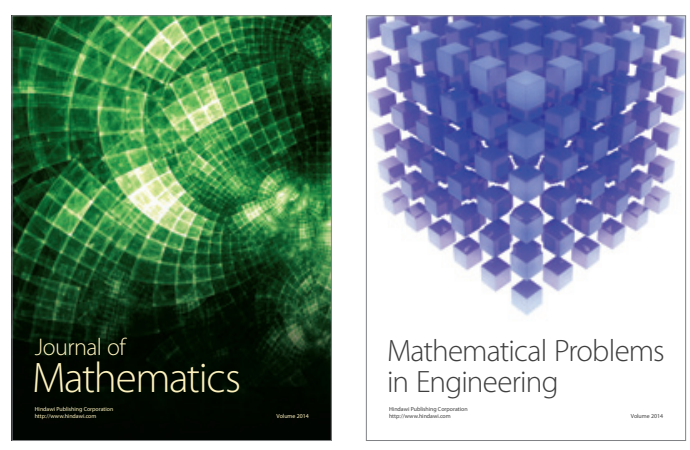

Mathematical Problems in Engineering
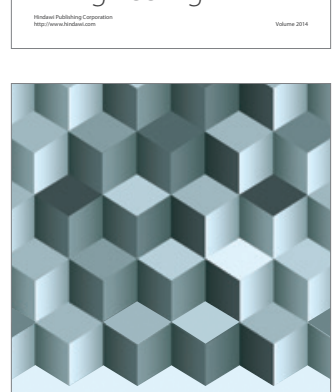

Journal of

Function Spaces
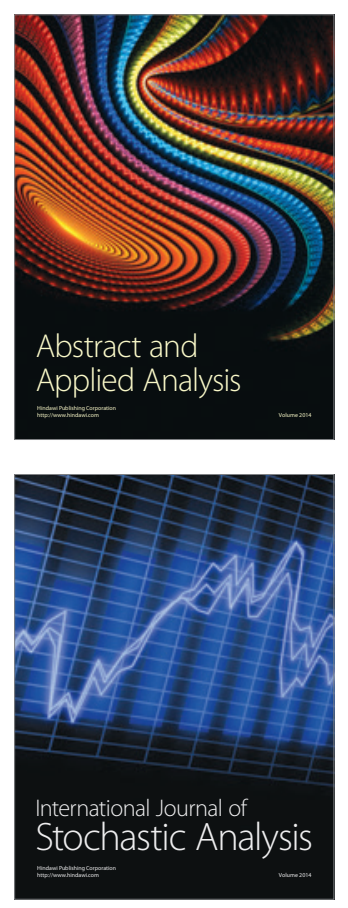

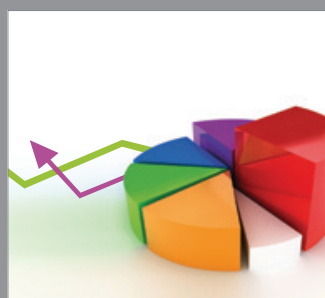

ournal of

Probability and Statistics

Promensencen
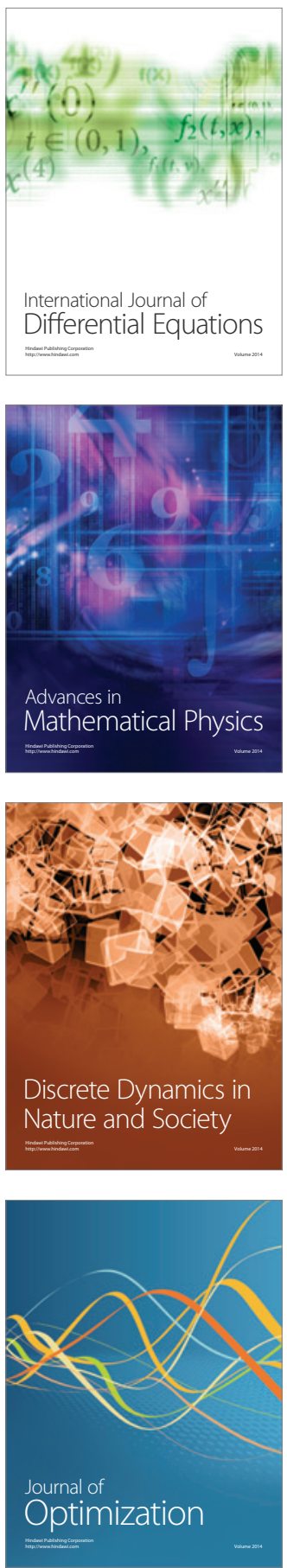